Journal of

Synchrotron

Radiation

ISSN 0909-0495

Received 14 January 2010

Accepted 17 September 2010

\section{Indirectly illuminated X-ray area detector for $X$-ray photon correlation spectroscopy}

\author{
Yuya Shinohara, ${ }^{\mathrm{a}, \mathrm{b} *}$ Ryo Imai, ${ }^{\mathrm{a}}$ Hiroyuki Kishimoto, ${ }^{\mathrm{c}}$ Naoto Yagi ${ }^{\mathrm{d}, \mathrm{b}}$ and \\ Yoshiyuki Amemiya ${ }^{a, b}$
}

${ }^{a}$ Graduate School of Frontier Sciences, The University of Tokyo, 5-1-5 Kashiwanoha, Kashiwa, Chiba 277-8561, Japan, ${ }^{\mathbf{b}}$ CREST/JST, Japan, ' Sumitomo Rubber Industries, 2-1-1 Tsutsui, Chuo, Kobe, Hyogo 651-0071, Japan, and dJASRI/SPring-8, 1-1-1 Kouto, Hyogo 679-5198, Japan. E-mail: yuya@k.u-tokyo.ac.jp

\begin{abstract}
An indirectly illuminated X-ray area detector is employed for X-ray photon correlation spectroscopy (XPCS). The detector consists of a phosphor screen, an image intensifier (microchannel plate), a coupling lens and either a CCD or CMOS image sensor. By changing the gain of the image intensifier, both photoncounting and integrating measurements can be performed. Speckle patterns with a high signal-to-noise ratio can be observed in a single shot in the integrating mode, while XPCS measurement can be performed with much fewer photons in the photon-counting mode. By switching the image sensor, various combinations of frame rate, dynamic range and active area can be obtained. By virtue of these characteristics, this detector can be used for XPCS measurements of various types of samples that show slow or fast dynamics, a high or low scattering intensity, and a wide or narrow range of scattering angles.
\end{abstract}

Keywords: indirectly illuminated X-ray detectors; X-ray photon correlation spectroscopy.

\section{Introduction}

X-ray photon correlation spectroscopy (XPCS) is a method of measuring the temporal intensity fluctuations of a speckle pattern caused by changes in the spatial arrangement of the scatterers in a sample. This method has attracted considerable attention as a promising tool for investigating the slow microscopic dynamics of various samples (Grübel et al., 2008). To perform XPCS experiments, both (partially) coherent $\mathrm{X}$-rays and a highly sensitive X-ray detector with high spatial and temporal resolutions are required. Various types of area detectors are now used for XPCS measurements, such as the Medipix2 detector (Carrona et al., 2008), the PILATUS detector (Westermeier et al., 2009) and the directly illuminated CCD (Livet et al., 2000). Among these detectors, the directly illuminated CCD is widely used in synchrotron facilities. Photon-counting detectors and integrating detectors in the photon-counting mode are desirable in XPCS measurement to maximize the signal-to-noise ratio so that the radiation damage to the sample is minimized, which is a serious problem in many cases, particularly in application to soft materials. Such a measurement, however, requires a high time resolution because the number of photons per pixel per acquisition/ accumulation time should be less than unity to count the number of photons without photon pile-up problems.

The use of an indirectly illuminated area detector offers an alternative approach to XPCS measurement. By combining an image sensor, such as a CCD or CMOS (complementary metal-oxide semiconductor), with an image intensifier, various combinations of gain, frame rate, active area and dynamic range can be obtained. For example, by changing the gain of the image intensifier, both the measurement in the photoncounting mode and that in the integrating mode, which leads to speckle patterns being obtained with a wide dynamic range, can be performed, while the frame rate and dynamic range can be easily chosen by changing the type of image sensor. Indirectly illuminated area detectors play an important role in many X-ray scattering experiments with synchrotron radiation (Gruner et al., 2002). There are mainly three types of indirectly illuminated area detectors. One detector uses fiber optics to couple a phosphor screen with an image sensor (Allinson, 1994). This detector is widely used in diffraction experiments, particularly protein crystallography experiments. Another detector uses optical relay lenses to couple a phosphor screen with an image sensor. This detector has a high spatial resolution and is mainly used in imaging experiments. The sensitivity of these two types of detectors is not sufficiently high for performing XPCS measurements because the intensity of the signal resulting from a single $\mathrm{X}$-ray photon generally does not exceed that of the noise of the image sensor. The use of an image intensifier offers the possibility of overcoming this issue (Amemiya et al., 1995). In this article, an application of such an indirectly illuminated detector to XPCS measurement is described. 


\section{Experimental details}

\subsection{Materials and XPCS setting}

As a sample for XPCS measurement, we used commercialgrade carbon black, which was added to styrene butadiene rubber (SBR) with a volume fraction of $10 \%$. The thickness of the sample was $1 \mathrm{~mm}$. XPCS experiments were carried out at BL40XU, SPring-8 (Japan). The XPCS setting in this beamline is described elsewhere (Shinohara et al., 2007). The light source of this beamline is a helical undulator, which provides highly intense quasi-monochromatic X-rays without a monochromator (Inoue et al., 2001). The flux density of X-rays was approximately $10^{17}$ photons $\mathrm{s}^{-1} \mathrm{~mm}^{-2}$ and the spectral resolution of the X-rays, $\Delta \lambda / \lambda$, was $1.8 \times 10^{-2}$, where $\lambda$ represents the X-ray wavelength. This value was sufficiently small for performing XPCS experiments in a small-angle scattering region (Shinohara et al., 2007). The intensity of X-rays was adjusted by changing the size of the front-end slits, which also changed the spatial coherence length; however, this did not affect the result in the present study and is not discussed here. The X-ray energy in this study was $10.5 \mathrm{keV}$ and the distance between the sample and detector was approximately $3 \mathrm{~m}$. A partially coherent X-ray beam was produced by inserting a pinhole $(5 \mu \mathrm{m}$ diameter) made of tantalum $15 \mathrm{~cm}$ upstream of the sample. Parasitic scattering was removed at another pinhole, which was installed slightly upstream of the sample. The FWHM size of the X-ray beam at the sample position was measured by the knife-edge method to be $5.3 \times 4.8 \mu \mathrm{m}$ $(\mathrm{H} \times \mathrm{V})$.

\subsection{Indirectly illuminated $X$-ray area detector}

An indirectly illuminated X-ray area detector was specially manufactured by Hamamatsu Photonics. A schematic view of the detector system is given in Fig. 1. It consists of (1) a beryllium entrance window, (2) an input phosphor screen ( $8 \mu \mathrm{m}$ thick) on a fiber-optic plate (FOP), (3) a 1:1 image intensifier composed of a photocathode and a microchannel plate (MCP), (4) optical relay lenses, and (5) an image sensor. For the phosphor, the fast-decay phosphor $\mathrm{P} 46\left(\mathrm{Y}_{3} \mathrm{Al}_{5} \mathrm{O}_{12}: \mathrm{Ce}\right)$

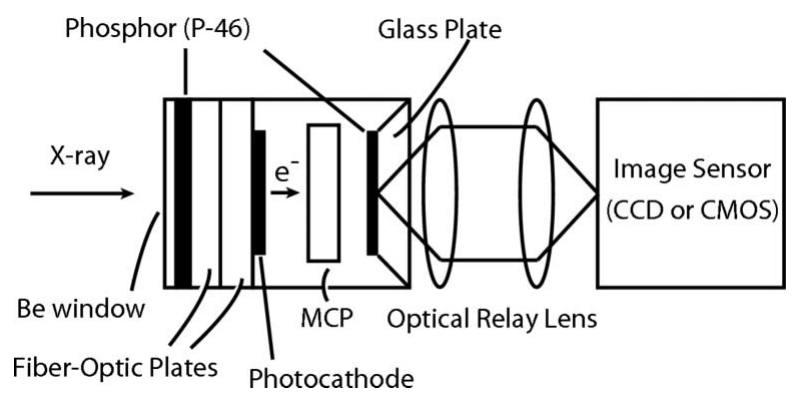

Figure 1

Configuration of an indirectly illuminated X-ray area detector for XPCS. The detector consists of (1) a beryllium entrance window, (2) an input phosphor screen on a fiber-optic plate, (3) an image intensifier [a photocathode and a microchannel plate (MCP)], (4) two optical relay lenses, and (5) an image sensor. An X-ray image on the phosphor screen is intensified and focused on the image sensor. The CCD and CMOS image sensors are easily exchanged by using a Philips mount.
Table 1

Principal characteristics of the interline-transfer CCD and fast-CMOS.

\begin{tabular}{|c|c|c|}
\hline & CCD (C4880-80) & CMOS \\
\hline Format & $656 \times 494$ & $1024 \times 1024$ \\
\hline Catalog pixel size & $9.9 \mu \mathrm{m} \times 9.9 \mu \mathrm{m}$ & $20 \mu \mathrm{m} \times 20 \mu \mathrm{m}$ \\
\hline Maximum frame rate (frames $\left.\mathrm{s}^{-1}\right) \dagger$ & 28 & 5400 \\
\hline Catalog readout noise (r.m.s.) & $4 \mathrm{e}^{-}$ & - \\
\hline
\end{tabular}

$\dagger$ Without binning and sub-array readout.

Table 2

Characteristics of the image intensifier.

\begin{tabular}{llll}
\hline Effective radius & Maximum gain & Resolution & Photocathode \\
\hline $25 \mathrm{~mm}$ diameter & $6.8 \times 10^{3}$ & 64 line-pairs $\mathrm{mm}^{-1}$ & Multi-alkali \\
\hline
\end{tabular}

was used. The decay time is approximately $100 \mu$ s, which is suitable for time-resolved measurement. The input FOP consists of two successive FOPs at present. In this system an $\mathrm{X}$-ray image is first converted to an intensified visible-light image, which is then focused onto the image sensor by the optical relay lenses. The image sensor can be easily exchanged owing to the adoption of a mechanical attachment called a Philips mount and the optical relay lenses are designed to be easily exchanged. In this study, two types of image sensors were used: an interline CCD (C4880-80; Hamamatsu Photonics) and a fast CMOS (FASTCAM SA-1.1; Photron). The principal characteristics of the image sensors are listed in Table 1. The image intensifier is a key component of the system; its characteristics are shown in Table 2. The gain of the MCP can be easily adjusted by changing its voltage on the MCP. The exposure times were $36 \mathrm{~ms}$ for the CCD and $0.18 \mathrm{~ms}$ for the CMOS. The image was taken at $5 \mathrm{~Hz}$ (integrating mode) or $28 \mathrm{~Hz}$ (photon-counting mode) for the CCD and $5400 \mathrm{~Hz}$ for the CMOS. The dimensions and weight of the box containing the image intensifier are $25(\mathrm{~W}) \times 40(\mathrm{D}) \times 30$ (H) $\mathrm{cm}$ and approximately $10 \mathrm{~kg}$, respectively.

\subsection{Data acquisition and processing}

Two types of image sensors were used: a fast CMOS and an interline CCD. Speckle images were first recorded on a hard disk and then were processed after the measurement. A detailed description of data processing can be found in a previous paper (Lumma et al., 2000). The method of data storage and the maximum number of images in a single sequence depend on the type of image sensor. In the case of the CMOS, the analogue signal was digitized by a 12-bit analogue-to-digital (A/D) converter. The obtained sequence of data was first recorded on random access memory (RAM) in the CMOS itself and then transferred to storage devices via an ethernet cable. In this case the maximum number of images taken in a single sequence (5400 images) is limited by the size of the RAM ( $8 \mathrm{~GB})$. It can also be increased by binning and sub-pixel readout.

In the case of the CCD, the measurement software HiPic (Hamamatsu Photonics) was used to record the images. The analogue signal was digitized by a 12-bit $\mathrm{A} / \mathrm{D}$ converter and 
was transferred to a frame grabber on a personal computer. We chose two modes of recording: one mode was used to record the images on a RAM and the other mode was used to record the images on a hard disk using the Hamamatsu Image Sequence (HIS) mode. When we record the images on RAM, the maximum number of images is limited by the size of the RAM (4 GB) in the present study. On the other hand, the maximum number is limited by the size of the hard disk when we use the HIS mode. In this case, the rate of image acquisition is limited by the time required to transfer and record data on the disk. We confirmed that the acquisition of up to 10000 images at a rate of $28 \mathrm{~Hz}$ with $36 \mathrm{~ms}$ exposure can be achieved in the HIS mode.

For both types of image sensors, external triggering is possible. Furthermore, the high voltage on the MCP can be rapidly switched on and off, which makes a sub-millisecond external gate mode feasible.

\section{Results and discussion}

\subsection{Integrating mode}

Fig. 2(a) shows a raw X-ray scattering image of carbon black aggregates in SBR taken using the CCD. When the incident flux had high intensity, a speckle image was obtained in a single exposure of $36 \mathrm{~ms}$. The result of the calculation of the ensemble-averaged intensity correlation function using 2500 frames is shown in Fig. 3. The numbers of pixels contributing to the average were $181,214,237,283$ and 331 for $q=|\mathbf{q}|=$ $0.023,0.028,0.033,0.038$ and $0.043 \mathrm{~nm}^{-1}$, respectively, where the scattering vector $\mathbf{q}$ is defined as the difference between the wavenumber vectors of incident and scattered X-rays. Two steps of relaxation, which are similar to those obtained for gels containing carbon black (Trappe et al., 2007), were observed. The dependence of relaxation time $\tau$ on $q$ was obtained for carbon black in rubber, as shown in Fig. 4. The result shows a scaling of $\tau \simeq q^{-1}$, which is common for many dense systems (Cipelletti et al., 2003). A detailed discussion on these behaviors will be presented elsewhere. In the following, we focus on the characterization of the detector. From Fig. 3, it is evident that the autocorrelation function $g^{(2)}(t)$ decays from approximately 1.03 to approximately 1.00 for delay times exceeding $100 \mathrm{~s}$. The value of $g^{(2)}(t \rightarrow 0)$ (speckle contrast) is small compared with the ideal value of $g^{(2)}(t=0)=2$. This speckle contrast depends on the values of the scattering vector, sample thickness and so forth (Abernathy et al., 1998). Following previous studies, we calculated the theoretical value by considering the transverse and longitudinal coherent lengths, the beam size and the sample thickness $(1 \mathrm{~mm})$, and we confirmed that measured contrast is from one-third to onehalf of the calculated contrast. Two factors are thought to be (a)

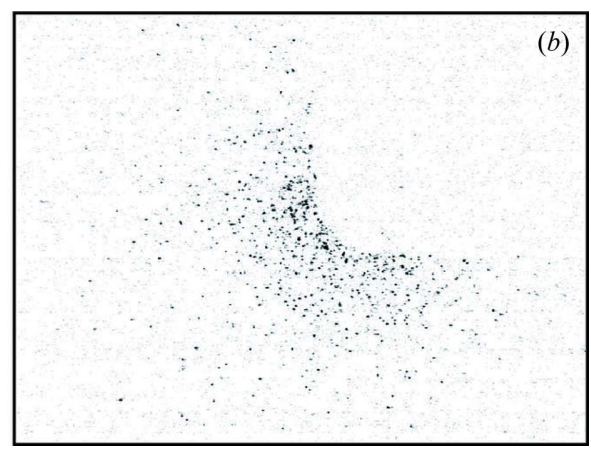

(b)

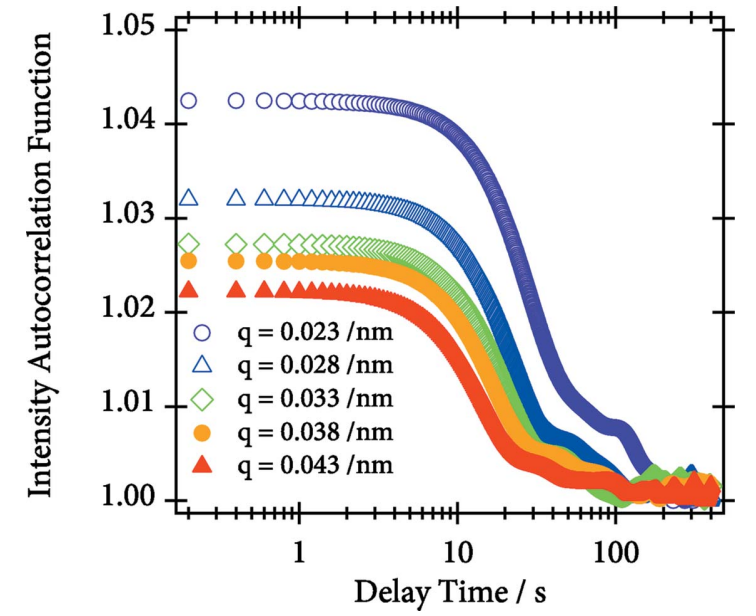

Figure 3

Normalized intensity autocorrelation function obtained from scattering of carbon black in rubber, derived from 2500 images.

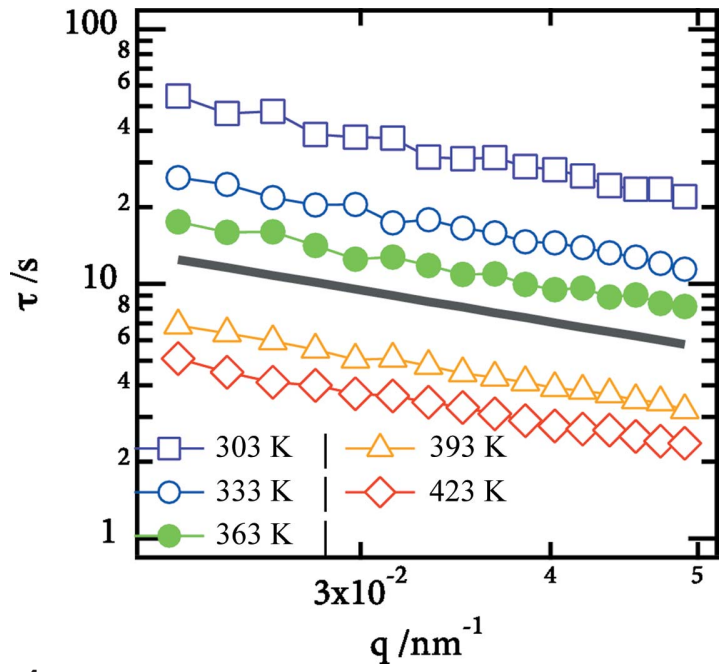

Figure 4

The $q$-dependence of initial relaxation time as an example of XPCS measurement with the present system. The solid line shows $\tau \simeq q^{-1}$, which indicates that carbon black shows the ballistic motion characteristic of dense systems. As the temperature increases, relaxation becomes faster. 
the source of the discrepancy: the smearing produced by the detector resolution and the existence of faster dynamics. We briefly discuss the degree of contrast reduction owing to the spatial resolution of the detector in the present study. The FWHM width of the point spread function (PSF) of the detector is estimated to be about $30 \mu \mathrm{m}$ from the knife-edge method. From the calculation, the radius of the speckle on the detector plane is estimated to be about $35 \mu \mathrm{m}$. By combining these results, the radius of the smeared speckle is calculated to be about $39 \mu \mathrm{m}$, which agrees reasonably well with the measured value of about $35-45 \mu \mathrm{m}$. Thus, the contrast decreases to approximately $90 \%$ of the original value. The main factor causing the spread of the PSF originates from the $\mathrm{MCP}$, and an improvement in the spatial resolution of the MCP is required to increase the speckle contrast.

In integrating measurement mode, the dynamic range of the detector is an important factor. Here, we define the dynamic range as

$$
\text { Dynamic range }=\frac{I_{\max }-D_{\text {avg }}}{\sigma_{\mathrm{s}}},
$$

where $I_{\max }, D_{\text {avg }}$ and $\sigma_{\mathrm{s}}$ in analog-digital units (ADUs) are the bit depth of the image sensors (12 bit $=4096)$, the intensity of the dark image averaged over the images, and the standard deviation of the dark images, respectively. By using the values of noise calculated in the following section, the dynamic ranges of the CCD and CMOS are estimated to be 1460 and 670 , respectively. Thus, the quality of images is sufficiently high for use in many types of XPCS experiments. The dynamic range can be expanded further by using other detectors with a higher quality, such as a full-frame transfer $\mathrm{CCD}$ at the expense of time resolution.

\subsection{Photon-counting mode}

The detector was also used in the photon-counting mode. A CCD image with a low incident X-ray flux is shown in Fig. 2(b), where the exposure time was $36 \mathrm{~ms}$. In this case the number of photons that were incident on the detector was approximately 100 . The signal from an individual photon is visible, as clearly seen in the figure. The detector with the CMOS sensor gives the same type of image.

To evaluate the performance of detectors in the photoncounting mode, we investigated the amount of noise and the signal produced by a single $\mathrm{X}$-ray photon by considering the photon-sharing problem. First, we evaluated the noise of the image sensors. In the absence of incident X-rays we obtained a dark image that mainly contained two types of noise: a timeindependent offset voltage, which was introduced in the A/D process, and a time-dependent dark current in the image sensors. As this dark image is added to a real signal in a measured image, it needs to be subtracted from the measured image before data processing. When we performed XPCS experiments, the effect of dark current on the data quality was generally negligible because the image accumulation time was short, typically less than $100 \mathrm{~ms}$. Thus, we ignored the timedependent component of the dark image. To reduce the fluc- tuation of introduced noise by dark-image subtraction, the average of more than 5400 dark images was obtained. The averaged dark image represents the offset components of the dark image. When the spatial variation of intensity was ignored, the averaged dark noise levels were calculated to be 633.2 and 581.9 for the CCD and CMOS in ADUs, respectively. Note that these averaged dark noise levels in ADUs cannot be directly compared between the two sensors and that the above result does not mean that the noise level of the CMOS is lower than that of the CCD. To evaluate the amounts of intensity fluctuations in dark images, we calculated the standard deviations of dark images at each pixel, $\sigma_{\mathrm{s}}(\mathbf{r})$, where $\mathbf{r}$ represents the position of the pixel. The standard deviations of the readout noise, $\sigma_{\mathrm{s}}=\sum_{\mathbf{r}} \sigma_{\mathrm{s}}(\mathbf{r}) / N_{\text {pix }}$, which was averaged over the entire image, were 2.37 for the CCD and 5.20 for the CMOS in ADUs from calculations using the 5400 images. Here, $N_{\text {pix }}$ represents the total number of pixels of the image sensor. The noise was well fitted with a Gaussian function, although there was an exponential tail corresponding to a few hot pixels, as shown in Fig. 5 (lower panel).

The charge-sharing problem needs to be considered when we perform measurements in the photon-counting mode. Because the FWHM of the PSF of this detector is larger than the pixel size, the local response to an individual photon is always shared by multiple pixels. In this case the binning of pixels solves the charge-sharing problem. To evaluate the effect of binning on the data quality, $\sigma_{\mathrm{s}}$ was calculated for the dark images with $2 \times 2$ and $3 \times 3$ binning, as shown in Fig. 5 . The values of $\sigma_{\mathrm{s}}$ for binned CCD images were 1.5 and 1.1 with $2 \times 2$ and $3 \times 3$ binning, and those for the CMOS were 2.87 and 2.08 with $2 \times 2$ and $3 \times 3$ binning. Note that the binning was processed by software, not on hardware. Binning on hardware would reduce the value further.

To perform measurements in the photon-counting mode using area detectors, the local responses to individual X-ray photons need to be isolated from each other. For this purpose the droplet algorithm (Livet et al., 2000) has been widely used. By using this algorithm we calculated the total intensity of each droplet produced by a single photon. In this algorithm a lower threshold on the charges accumulated in a pixel was defined. Neighboring pixels charged above this threshold were considered as belonging to the same droplet. In this paper we considered the spread of charge by including all adjacent pixels in the definition of the droplets, and the threshold was set to $5 \sigma_{\mathrm{s}}(\mathbf{r})$ at each pixel. The total intensity and area of each droplet were then calculated. The calculation showed that the averaged areas of a single droplet were 9.7 and 5.5 pixels for the CCD and CMOS, respectively, without binning. Thus, we binned each image and compared the results obtained with binning with those obtained without binning. The histograms of droplet intensities for the CCD and CMOS are shown in Figs. 6 and 7, respectively. It is clear that the intensity of each droplet was well above the threshold $5 \sigma_{\mathrm{s}}$ and that the measurement in the photon-counting mode was successfully performed with this detector. For the data obtained without binning, the profiles show oscillation owing to photon sharing. The oscillation originates from the fact that only the pixels 

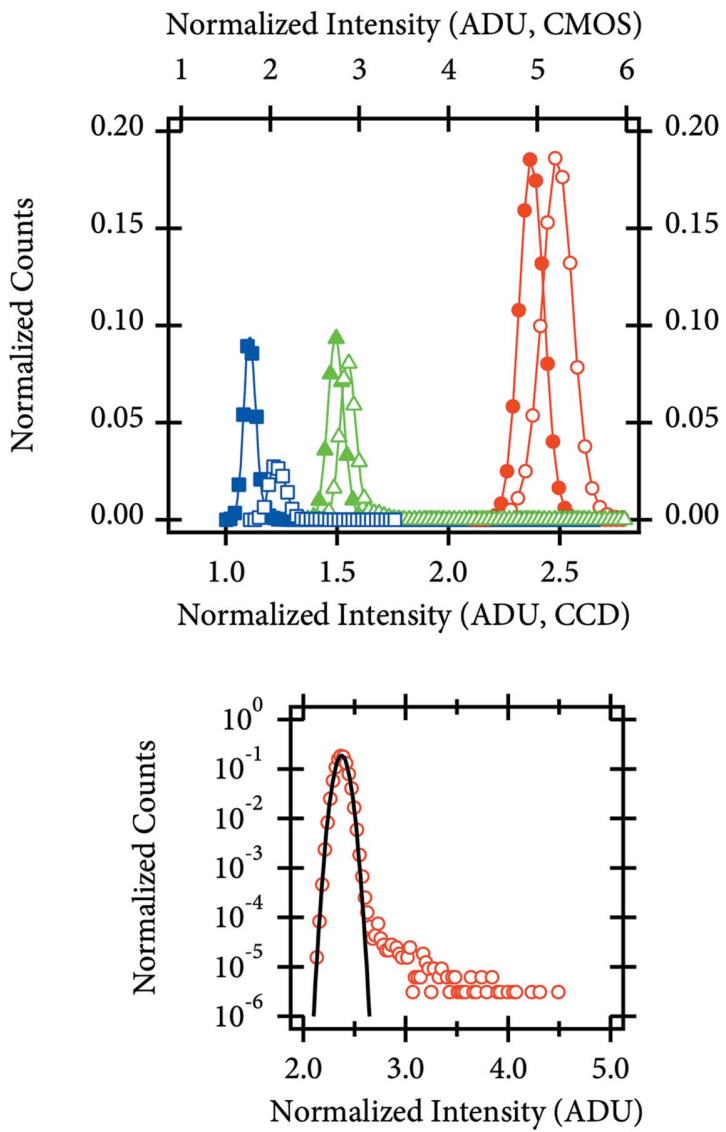

Figure 5

(Top) Distribution of the standard deviation of dark noise, which is averaged over dark images. Closed and open symbols correspond to the results for the CCD and CMOS, respectively (circles: without binning; triangles: $2 \times 2$ binning; squares: $3 \times 3$ binning). To compare the different configurations, both abscissas and ordinates are normalized. The abscissas are normalized by the number of pixels in a bin. The ordinates are normalized by the number of pixels in a single frame. (Bottom) Semi-log plot of the result for the CCD without binning. The solid line indicates the fitting result with a Gaussian function.

whose intensity is larger than the threshold are considered to belong to a droplet. When binning is not performed, only the center or nearest-neighboring pixels are considered to belong to a droplet, and the intensity of the neighboring pixels, which is smaller than the threshold, is not considered in the calculation of droplet intensity. This discussion is based on the fact that the interval between peaks agrees with the threshold value of $5 \sigma_{\mathrm{s}}$. It is confirmed that the interval between peaks corresponds to the threshold value by analyzing data with different threshold values, which is omitted in the present paper. The oscillation vanishes when binning is carried out. It should be noted that the number of X-ray photons incident on the detector is different between the CCD $(\sim 100)$ and CMOS $(\sim 10)$. This difference leads to the different ordinates in Figs. 6 and 7.

One drawback of the present system is that the droplet area is large: 9.7 and 5.5 pixels for the CCD and CMOS, respectively, without binning. This large droplet area is associated with the use of indirectly illuminated area detectors. In the present system the spread of the droplet originates from the

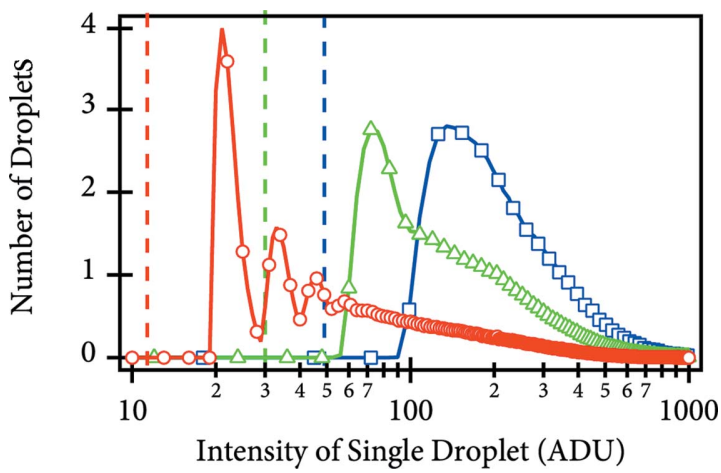

Figure 6

Distribution of the intensity of a single droplet in ADUs per frame for the CCD without binning (circles), with $2 \times 2$ binning (triangles) and with $3 \times 3$ binning (squares). The dashed lines show the values of $5 \sigma_{\mathrm{s}}$ for the data without binning (red), with $2 \times 2$ binning (green) and with $3 \times 3$ binning (blue).

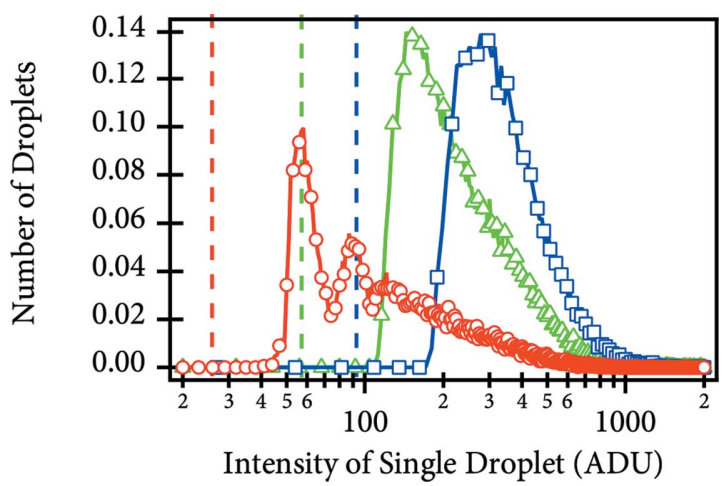

Figure 7

Distribution of the intensity of a single droplet in ADUs per frame for the CMOS without binning (circles), with $2 \times 2$ binning (triangles) and with $3 \times 3$ binning (squares). The dashed lines show the values of $5 \sigma_{\mathrm{s}}$ for the data without binning (red), with $2 \times 2$ binning (green) and with $3 \times 3$ binning (blue).

fact that the spread of the PSF of the system, particularly that of the MCP, is large compared with the pixel size. This large droplet area of a single photon induces the pile-ups even with low-incident X-rays, and the total flux in the detector per image should be less than $10^{2}$. Nevertheless, this does not cause any serious problem in many cases in which the radiation damage is a dominant factor limiting the incident X-ray flux.

\section{Summary}

An indirectly illuminated X-ray area detector has been employed for XPCS measurements. By using the detector in the integrating mode, speckle patterns were successfully observed in a single shot and the XPCS measurement of filled rubber at $5 \mathrm{~Hz}$ was demonstrated. When an intense X-ray is obtained and the effect of radiation damage on the XPCS data can be neglected, the measurements in the integrating mode are suitable for performing XPCS. In the photon-counting mode, signals from a single photon were observed. When the scattering intensity is not sufficiently high for observing speckle patterns in a single shot with appropriate exposure times, the photon-counting mode is vital for XPCS measure- 
ments. This detector can be operated in both of these modes; thus, it can be used for XPCS measurements at a variety of beamlines and for various kinds of samples. Owing to its flexibility, the detector can be used not only for XPCS measurements but also for other scattering experiments, such as high-resolution diffraction experiments with a high sensitivity.

The authors thank Dr N. Ohta (JASRI) and the members of the Amemiya Laboratory (University of Tokyo) for their kind support in the XPCS experiments. The XPCS experiments were performed under the approval of the JASRI programming committee (Proposal Nos. 2008B1153 and 2009A1182).

\section{References}

Abernathy, D. L., Grübel, G., Brauer, S., McNulty, I., Stephenson, G. B., Mochrie, S. G. J., Sandy, A. R., Mulders, N. \& Sutton, M. (1998). J. Synchrotron Rad. 5, 37-47.

Allinson, N. M. (1994). J. Synchrotron Rad. 1, 54-62.

Amemiya, Y., Ito, K., Yagi, N., Asano, Y., Wakabayashi, K., Ueki, T. \& Endo, T. (1995). Rev. Sci. Instrum. 66, 2290-2294.
Carrona, C., Chushkin, Y., Madsen, A. \& Cupane, A. (2008). Phys. Rev. Lett. 100, 055702.

Cipelletti, L., Ramos, L., Manley, S., Pitard, E., Weitz, D. A., Pashkovski, E. E. \& Johansson, M. (2003). Faraday Discuss. 123, 237-251.

Grübel, G., Madsen, A. \& Robert, A. (2008). Soft-Matter Characterization, edited by R. Borsali \& R. Pecora, pp. 954-995. Heidelberg: Springer.

Gruner, S. M., Tate, M. W. \& Eikenberry, E. F. (2002). Rev. Sci. Instrum. 73, 2815.

Inoue, K., Oka, T., Suzuki, T., Yagi, N., Takeshita, K., Goto, S. \& Ishikawa, T. (2001). Nucl. Instrum. Methods Phys. Res. A, 467-468, 674-677.

Livet, F., Bley, F., Mainville, J., Caudron, R., Mochrie, S. G. J., Geissler, E., Dolino, G., Abernathy, D., Grübel, G. \& Sutton, M. (2000). Nucl. Instrum. Methods Phys. Res. A, 451, 596-609.

Lumma, D., Lurio, L. B., Mochrie, S. G. J. \& Sutton, M. (2000). Rev. Sci. Instrum. 71, 3274-3289.

Shinohara, Y., Kishimoto, H., Maejima, T., Nishikawa, H., Yagi, N. \& Amemiya, Y. (2007). Jpn. J. Appl. Phys. 40, L300-L302.

Trappe, V., Pitard, E., Ramos, L., Robert, A., Bissig, H. \& Cipelletti, L. (2007). Phys. Rev. E, 76, 051404.

Westermeier, F., Autenrieth, T., Gutt, C., Leupold, O., Duri, A., Menzel, A., Johnson, I., Broennimann, C. \& Grübel, G. (2009). J. Synchrotron Rad. 16, 687-689. 